\title{
Ultrasonic Sensors used to Detect the Position of the Mobile Service Robot
}

\author{
Ondrej Juruš ${ }^{1, *}$, Peter Ferenčík ${ }^{2}$ \\ Technical University of Košice, Faculty of Mechanical Engineering, Letná 9, 04200 Košice, Slovakia \\ Robert Bosch GmbH, Powertrain Solutions, Robert-Bosch-Straße 2, 71701 Schwieberdingen, Germany
}

\begin{abstract}
The article talks about our design of ultrasound-based localization system. The designed system is primarily intended for mobile robotics. The second part of this article is focused on the initial verification of the basic characteristics and accuracy of the position measurement. This location system is currently still in the process of improving the functions required for usage in mobile robotics. We are currently working on removing some types of interferences.
\end{abstract}

Keywords: mobile, robot, ultrasound, location, localization

\section{Introduction}

Achieving compromise between price and technical capabilities of a mobile robot is a task for everybody who sets out to create a device, able to compete with existing simple solutions. To develop economical solution, one is forced to choose from a wide range of technologies the one, which provides the best ratio of price versus offered technological capabilities. $[8,10]$ For our objectives, we have chosen ultrasound sensors. Advantages of ultrasound sensors are reasonable price, simple processing of the measuring signal and, in comparison with optical sensors as cameras, it is not susceptible to poor light conditions. But as with every technology, there is also a downside. The biggest disadvantage is susceptibility to temperature and humidity of the environment. Also, high quantity of rough particles of dust in the environment that can cause reflection of soundwaves can distort and degrade the measuring. $[2,5]$ However, if we focus on ordinary places that mobile robots use for ancillary operations for production, like storage spaces and service spaces for assembly workers and service personnel, it is obvious that foulness of environment is a minimal and relatively negligible problem. Mobile robots are tasked with distribution of material in workspaces and organization of storage spaces. For simple operation and orientation, induction or optical principles are often used - a track is marked on the floor, which corresponds with the trajectory of its movement. $[1,6]$ These variants have another downside - robots, or service vehicles, are forced to move one after another in these tracks, which causes slowing down of vehicles on the same route (Fig. 1). This problem is either completely unhandled, or when the delay is serious, the operator has to invest sizeable sums to solve this problem.

On this account, using robot localization by ultrasound transmitters enables us to control multiple robots simultaneously, and allows us to prevent these problems by choosing a different trajectory for the robot, or overtaking a slower robot. In behalf of the common position system it is possible to coordinate and control the robots jointly and thus allowing increase of efficiency of robot movement. [11,12]

\section{Principle of distance measurement using an ultrasound}




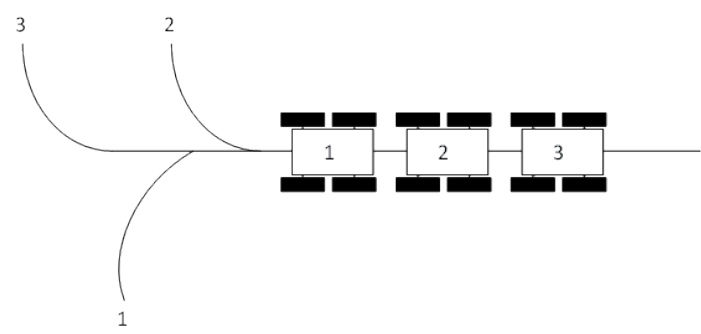

Figure 1: Delay of material flow in consequence of the joint track of robots

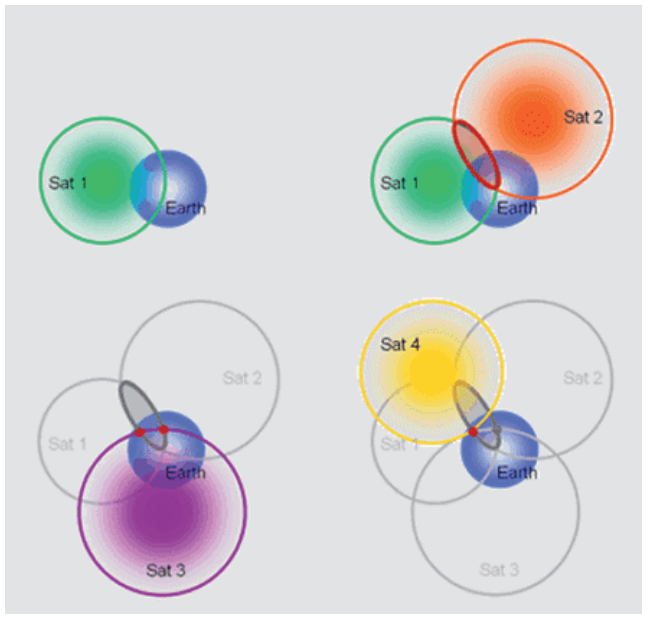

Fig. 2 Basic principle of trilateration used in GPS [3]

to focus on the way in which the robots are set within the workspace. Tracking a robot moving in a space is only possible if each transmitter has clearly defined coordinates. These coordinates must relate to the coordinate system of the room. $[9,10,13]$ This system is regarded as the global coordinate system of the room (GCSR). The robot has its own coordinate system, which we will call a coordinate system of the robot (CSR). [6,7] For the localization and control of the robot we will choose from several different principles of robot positioning. To determine the current location of the robot, there is the possibility to choose a simple and well-tried method - trilateration - by use of measuring the distance between the transmitter and receiver, which is defined by the length of time, in which the sound signal travels between the transmitter and receiver. The speed of the ultrasound signal is affected by humidity and temperature of the environment. In the speed calculation, we count on standard humidity, in a closed room with the temperature at $20^{\circ} \mathrm{C}$ the humidity is $55 \%$ on average. The speed of sound in vacuum, denoted as $C_{0^{\prime}}$ is
$331.4 \mathrm{~ms}^{-1}$. The actual speed of sound $\mathrm{v}$ is, depended on temperature $T$ and this dependence can be roughly expressed by a linear relation

$v=c_{0}+0.61 \mathrm{dT}$.

where $v$ stands for real sound velocity and dT stands for temperature change from $0^{\circ} \mathrm{C}$.

\section{Design of localization system}

Due to the knowledge of the distance between the transmitter and receiver, we can use the basic principle of trilateration. A general location of the robot is deduced thanks to the relations between the coordinate system of the robot and the global coordinate system of the room. To calculate the position of the robot, we must be aware of the position of the transmitters about the origin of GCSR (Fig. 3). Distances to the origin of GCSR are expressed with the vectors $d_{0}$ and $d_{1}$. If we know the range from the transmitters to the origin of GCSR, and if we measure the distance between the robot and each transmitter, which are defined with the vectors $d_{0}$ and $d_{1}$, we can conclude the distance between the robot and the origin of GCSR. For the actual calculation we need to know several other parameters. One of the main parameters is the spacing of the transmitters and the shape of the transmitter placement. At the same time, it is necessary to treat measurement errors. One of the ways to eliminate faulty measurements is to define the minimum possible distance.

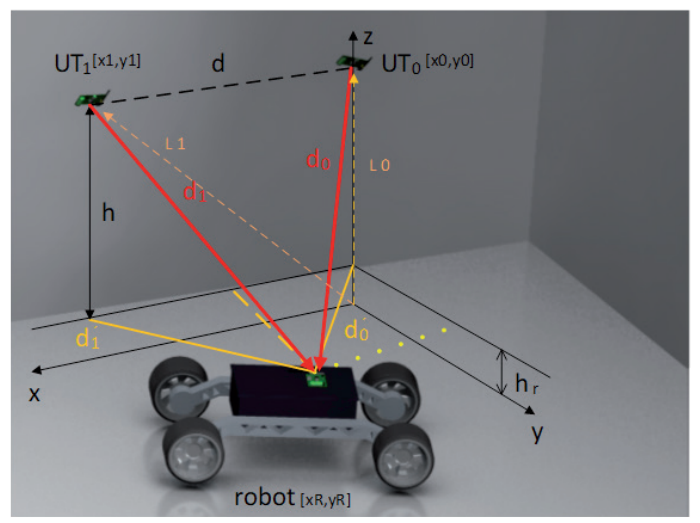

Fig. 3 Relation of robot coordinates and system coordinates

This will prevent stray signals or rebounds. These signals could cause serious measurement corruption and subsequently loss of the actual robot position. The basic idea of the transmitter placement is a 
regular triangle grid on the room ceiling. Opposite that, regular placement in a square grid of the transmitters allows for more accurate calculation of robot position. The essential minimum requirement to detect the robot location is to use three transmitters. For a more accurate measurement, a square grid was chosen, which allows cover all of the workspace with four transmitters.
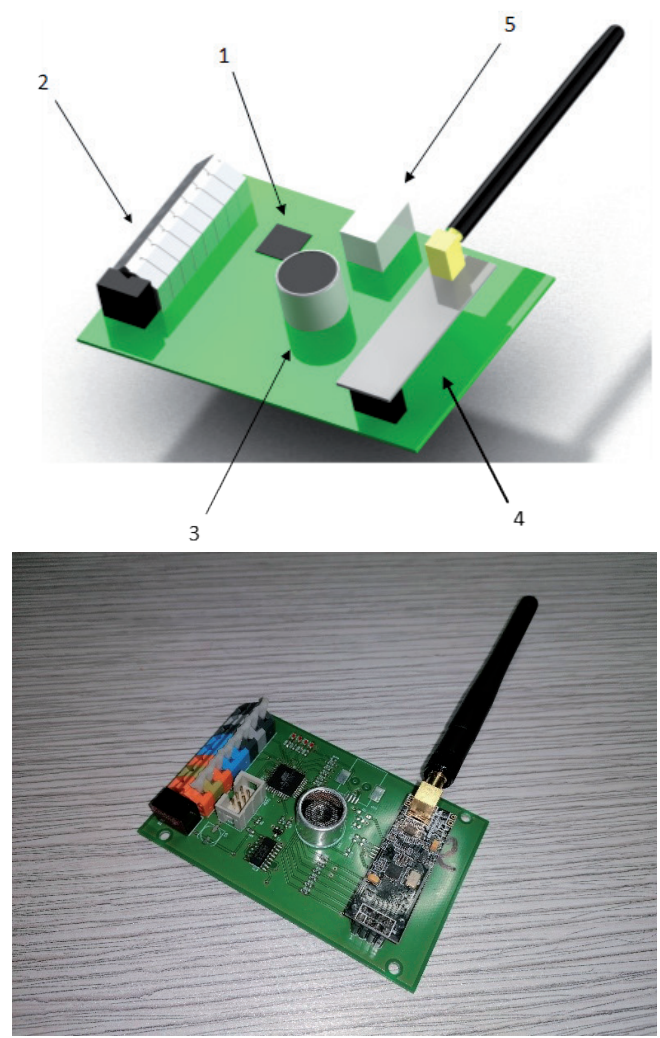

Fig. 4 Printed circuit board of the transmitter/receiver, (1) microcontroller, (2) connection clips, (3) transmitter/receiver, (4) communication antenna, (5) USB-B connector

\subsection{Measuring the accuracy of sensors}

In pursuit of high accuracy of localization, we were forced to get the properties of the measurement system and calibrate the system to minimize the deviations in measurements caused by the environment, rebounds, or other reasons. Printed circuit boards (Fig. 4) were designed and created for our needs, directly in our department. The basic measurement was to check the accuracy of the received signal. The measurement was implemented on a simple measuring frame, where we positioned the transmitter and receiver. For the control check we used a straight line equation:

distance $=0.1543 \cdot \operatorname{tact}+99.3140$

where 0.1543 is constant (distance travelled by an ultrasonic wave in $\mathrm{cm}$ ); tact is number of cycles counted by microcontroller; 99.3140 is dead distance of the ultrasonic sensor in $\mathrm{cm}$. Measurement was carried out with the software "Read_measure.exe". This program is developed and designed to measure the processor cycles and can calculate the distance between the transmitter and the receiver based on the relation in (2).

Table 1. Control check of the set value using cycles.

\begin{tabular}{|c|c|c|c|c|}
\hline $\begin{array}{l}\text { Measured values } \\
\text { number of cycles } \\
\text { counted by } \\
\text { microcontroller }\end{array}$ & $\begin{array}{l}\text { Multiplicity } \\
\text { number of } \\
\text { occurrences } \\
\text { in the } \\
\text { measured } \\
\text { sample }\end{array}$ & $\begin{array}{l}\text { Distance } \\
{[\mathrm{cm}]}\end{array}$ & $\begin{array}{l}\text { Average } \\
\text { measured } \\
\text { values }\end{array}$ & $\begin{array}{l}\text { Average } \\
\text { distance } \\
{[\mathrm{cm}]}\end{array}$ \\
\hline 520 & 2 & 179.55 & \multirow{10}{*}{523.713} & \multirow{10}{*}{180.120} \\
\hline 521 & 15 & 179.70 & & \\
\hline 522 & 29 & 179.86 & & \\
\hline 523 & 40 & 180.01 & & \\
\hline 524 & 18 & 180.17 & & \\
\hline 525 & 16 & 180.32 & & \\
\hline 526 & 9 & 180.48 & & \\
\hline 527 & 11 & 180.63 & & \\
\hline 528 & 9 & 180.78 & & \\
\hline 529 & 1 & 180.94 & & \\
\hline
\end{tabular}

The mentioned control check measurement was done at the room temperature of $18{ }^{\circ} \mathrm{C}$. The humidity of the environment was $57 \%$. The distance between the transmitter and receiver was set to $1778 \mathrm{~mm}$. Based on these parameters, the speed of sound in the environment was calculated using the relation in (1) as $349.56 \mathrm{~ms}^{-1}$. We carried out 150 measurements in these conditions. The acquired results with the use of software outputs are in Tab. 1. The average value was obtained as a sample mean define by the formula

$\bar{x}=\frac{1}{n} \sum_{i=1}^{n} x_{i}$.

Another important sample characteristic is the standard deviation. The standard deviation of the measured values is 0.316122 . The corresponding 
dispersion is 0.099933, obtained as

$$
s=\sqrt{\frac{\sum_{i=1}^{n}\left(x_{i}-x\right)^{2}}{n-1}} .
$$

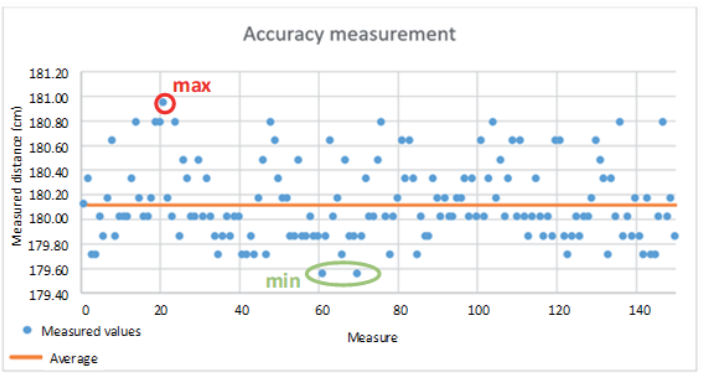

Fig. 5 Measurement of the accuracy of ultrasound sensor

The measured values and their real sequence over time are shown in Fig. 5. The expected average distance was $177.80 \mathrm{~cm}$ (not $180.12 \mathrm{~cm}$ ). It was established that a correction of parameters 0.1543 and 99.3140 is necessary in equation (2). As follows from the graph, the maximum deviation is $0.82 \mathrm{~cm}$ in the positive direction and 0.57 in the negative direction. The range is $1.39 \mathrm{~cm}$. By using cycles to time calculation, we get the ratio of cycles to milliseconds as 0.98. This error must be corrected in the future by calibrating the microcontroller clock. In our solution, an RC element is used as a clock circuit, which has not been calibrated and has this error.

Table 2. Control check of the set values using time

\begin{tabular}{|c|c|c|c|c|}
\hline $\begin{array}{l}\text { Measured values } \\
\text { number of cycles } \\
\text { counted by micro- } \\
\text { controller * } 0,98\end{array}$ & Multiplicity & $\begin{array}{l}\text { Distance } \\
{[\mathrm{cm}]}\end{array}$ & $\begin{array}{l}\text { Average } \\
\text { measured } \\
\text { values }\end{array}$ & $\begin{array}{l}\text { Average } \\
\text { distance } \\
{[\mathrm{cm}]}\end{array}$ \\
\hline 509.60 & 2 & 176.91 & \multirow{10}{*}{513.239} & \multirow{10}{*}{177.910} \\
\hline 510.58 & 15 & 176.99 & & \\
\hline 511.56 & 29 & 177.33 & & \\
\hline 512.54 & 40 & 177.67 & & \\
\hline 513.52 & 18 & 178.01 & & \\
\hline 514.50 & 16 & 178.35 & & \\
\hline 515.48 & 9 & 178.69 & & \\
\hline 516.46 & 11 & 179.03 & & \\
\hline 517.44 & 9 & 179.37 & & \\
\hline 518.42 & 1 & 179.71 & & \\
\hline
\end{tabular}

Using the constant 0.98, which tries to minimize square deviations and correct errors, we achieved the value of standard deviation 0.69893 and dispersion 0.4885 . The real range is $2.8 \mathrm{~cm}$.

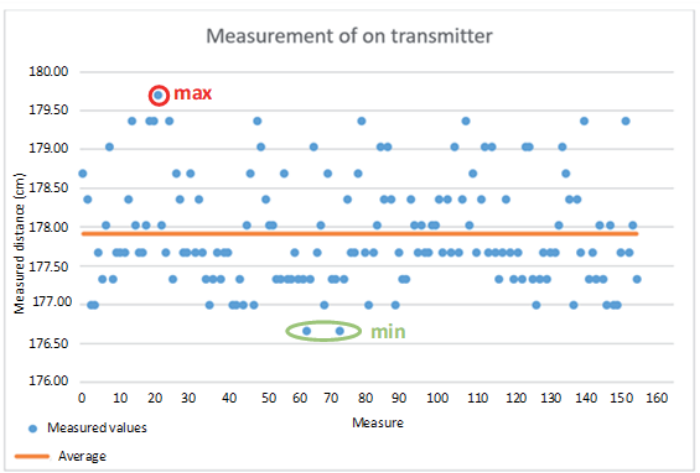

Fig. 6 Position measurement using one transmitter

By comparing multiplicities of measured values, we found that it is not an ideal Gauss curve. The peak is at 523 cycles. Using the time expression, it represents $512.54 \mathrm{~ms}$.

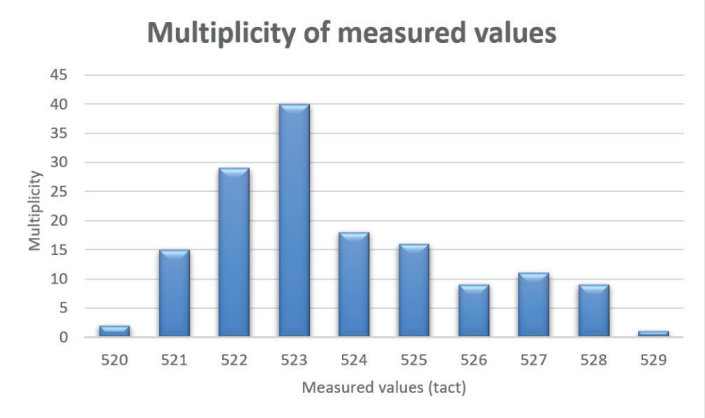

Fig. 7 Multiplicity of measured values

\section{Conclusions}

It is possible to detect a robot next to a wall with two receivers, since we have information about their coordinates and at the same time we have information that the robot can be situated only in one half of the scanned environment. However, if the robot is moving in an open space, it is necessary to utilize many more sensors than just 2. Thus, this system will be expanded into a larger system. A measuring grid will be used. The first steps will try to duplicate already existing systems. However, the goal is to develop original autonomous localization system. The next development will be the ability to detect the robot orientation. The main aim is to find mathematical relationships that will allow us to determine the position of the robot with 
respect to distributed transmitters. We measured these constants by placing the transmitter and receiver opposite to each other and repeating the measurement. We measured 150 values and tried to find mathematical dependence on the measured values. Using the line equation, we detected some existing indications, which values should be constant. The measurement used a program that measured the processor's clock. However, this is not a completely accurate measurement, taking into account the delays occurring in the generation of the signal, either mechanically (transmitting the transmitter diaphragm, generating a sufficient number of pulses) and at the same time from the programming point of view. These errors are seemingly insignificant from an overall perspective. However, taking into consideration the individual measurement steps and the mathematical deduction from the measured values, these seemingly negligible delays appear to be a serious aspect of the measurement. This means that these delays need to be considered. After obtaining relevant data from a single transmitter and receiver system, it is possible to extend this assembly to three and four transmitters. It is essential to verify the theory whether the three transmitters are sufficient to determine the exact position of the robot, or it is necessary to mount the transmitting grid using a regular grid consisting of four sensors. Validation of these theories is the basis for defining the environmental conditions to which this system can be applied. The system's present ideas must be subject to the capabilities that our positioning system is capable of providing.

\section{References and Notes}

1. Maciak M., Mizera I., et al. (2016). Regularization techniques in Joinpoint Regression. Statistical Papers, vol. 57, Issue 4, p. 939-955

2. Maciak M., et al. (2017). Testing Shape Constraints in Lasso Regularized Joinpoint Regression. AMIDAS 2015: Analytical Methods in Statistics, p. 105-122

3. GIS Commons: An Introductory Textbook on Geographic Information Systems giscommons.org

4. Karavaev, Y.L., Kilin, A.A., et al. (2016). Nonholonomic dynamics and control of a spherical robot with an internal omniwheel platform: Theory and experiments. Proceedings of the Steklov Institute of Mathematics, vol. 295, Issue 1, p. 158-167
5. Koniar, D., Hargas L., Hrianka M., et al. (2009). Measurement of Object Beating Frequency Using Image Analysis. International Conference on Applied Electronics Location: Pilsen

6. Duchon F. et al. (2012). Localization and navigation of mobile robots into the interior, Slovak edition, ISBN 978-80227-3646-6

7. Trebuna F., Pastor M., Liptak T., Mikova L., Virgala I., et al. (2016). An inspection of pipe by snake robot. International Journal of Advanced Robotic Systems. ISSN 1729-8814. vol. 13, p. 1-12

8. Gola A., Świć A., Design of storage subsystem of flexible manufacturing system using the computer simulation method, Actual Problems of Economics, 142 (4) 2013, pp. 312-318.

9. Kłosowski G., Gola A., Świć A., Application of Fuzzy Logic Controller for Machine Load Balancing in Discrete Manufacturing System, Lecture Notes in Computer Science, Vol. 9375, pp. 256-263, DOI: 10.1007/978-3-319-24834-9_31.

10. Rudawska A., Čuboňova N., Pomarańska K., Stanečková D, Gola A., Technical and Organizational Improvements of Packaging Production Processes, Advances in Science and Technology. Research Journal, Vol. 10, No. 30, 2016, pp. 182192. DOI: $10.12913 / 22998624 / 62513$.

11. Hajduk M., Semjon J., Jánoš R., Vagaš M., Robotics - Robotic Technique, et. al. 2015, Vol. 1, pp. 188. ISBN 978-880-5532500-2

12. Semjon J., Hajduk M., Sukop M., Baláž V. Pilat Z., Sulik Z., Putala J., Testing of parameters of proposed robotic wrist based on the precision modules. [et al.] - 2016. In: International journal of advanced robotic systems. Vol. 13 (2016), p. 1-7. - ISSN 1729-8814

13. Marcinko P., Semjon J., Baláž V., Vagaš M., Jánoš R., Proposal of Tracked Robot with Folding Arms / Peter Marcinko ... [et al.] - 2016. In: American Journal of Mechanical Engineering. Vol. 4, no. 7 (2016), p. 372-375. - ISSN 2328-4102 